\title{
Visualising the Multimedia Archives of Bourges's Institute of Electroacoustic Music
}

\author{
Florent Di Bartolo \\ University Paris-Est Marne-la-Vallée \\ Champs-sur-Marne, France \\ florent.dibartolo@u-pem.fr
}

\begin{abstract}
This paper describes the properties and functionalities of the application we have created to retrace the story and evolution of international competitions organised by the Bourges's Institute of Electroacoustic Music (IMEB) from 1973 to 2009 using a set of interactive data visualisations. This set of interactive data visualisations serve different purposes: it provides multiple views of the database it allows to browse but also gives insights about navigation paths that have been previously taken to assist their users in their browsing experience. This set of interactive data visualisations represent an attempt to give access to the content of a digital archive, not only by considering its main properties, but also by gathering information about its past exploration using multi-agent systems.
\end{abstract}

Digital humanities. Data visualisation. Database. Multi-agent systems. IMEB. Electroacoustic music.

\section{INTRODUCTION}

Bourges's Institute of Electroacoustic Music (IMEB) has been created in France in 1970 under the name "Groupe de musique électroacoustique de Bourges" (GMEB) by the composers Françoise Barrière and Christian Clozier who directed it until its closure in 2011. In 1994, the Bourges's Group of Electroacoustic Music became an Institute recognised by the French Ministry of Culture as a National Center of Musical Creation. During its forty years of existence this institute has been heavily involved in the promotion of electroacoustic music both on national and international scales. Its activities have included among others musical research, development of music-making software, creation of instruments and organisation of music festivals and competitions.

Artists all over the world have participated in the activities proposed by the IMEB. According to Christian Clozier, 274 artists from 43 countries have worked in their studios on 765 different audio compositions and 4,836 composers from 82 countries have presented their work during the competitions and music festivals they have organised starting from 1971.

From 2005 to 2011, the IMEB has donated all of its archives to the Bibliothèque nationale de France $(\mathrm{BnF})$ that is to say a complete set of multimedia data about the history of electroacoustic music and its worldwide diffusion.

In this paper, I will be describing part of the work that has been done to explore these archives as part of a research project directed by Geneviève Mathon and that has been financially supported by the Arts-H2H Labex. This paper focuses on the application that has been specially designed to retrace the story of the international electroacoustic music competitions organised by the IMEB from 1973 to 2009.

The application is presented as a prototype that can be accessed online (https://goo.gl/dlv07L). It includes a set of interactive data visualisations that provide information about the participants of the competitions organised by the IMEB and their works. To respect copyright laws, this online version doesn't give access to any audio records available at the National Library of France. Only the version of the application meant to be available at the $\mathrm{BnF}$ will grant such access. Despite these restrictions, we hope that the functionalities attached to the interactive data visualisations accessible online will be enough to understand how these audio compositions can be easily retrieved using the same set of functionalities and graphical interfaces. 


\section{VISUALISING MULTIMEDIA ARCHIVES}

To collect data about the international electroacoustic music competitions organised by the IMEB, multiple documents available at the $\mathrm{BnF}$ have been consulted including administrative forms and the minutes of each competition. Administrative forms provide information about the structure of the competitions itself and the evolving set of categories used to organise them. Minutes, on the other hand, contain information about each participant allowing us to know their name but also their current address and the number (or category) of each of their submissions. Some minutes contain also the titles of the audio works that have obtained a price allowing us to gather information about electroacoustic compositions whose quality has been publicly rewarded.

All this information has been entered in a database in order to be able to retrieve it using queries but also to be able to explore it using interactive data visualisations that provide overviews and allow its users to filter data (and retrieve more detailed information) based on user input. The data contained in this new database can also be used to create listings of award-winning pieces or statistics about the provenance of the competitions participants as those provided by Christian and Clozier Françoise Barrière. But these listings have the advantage to be dynamic. They respond to any change made to the relational database and can be linked, on different scales, to the data that have been used to generate them. The interactive visualisations presented below have been specially designed to look at the same data from different points of view. Each of them focuses on one property of the information that has been collected in order to be able to compare multiple views of the same data set and be able to perceive more easily its main properties and elements. To emphasise the multiplicity of points of view, each visualisation doesn't use the same type of structure nor provide the same functionalities.

\subsection{Flow Diagram}

A Sankey diagram has been used to represent the set of categories chosen by Françoise Barrière and Christian Clozier to structure the IMEB international competitions and sort out submissions (see Figure 1). It reveals the evolution of the competitions main categories from one edition to another. It permits its users to identify the number of categories used for each edition and their weight based on their longevity. It lets detect their reorganisation too with the appearance for example in 1988 of new categories called "Magister," "Residency" and "Quadrivium" that regroup subcategories that have been previously used to separate different types of submissions.

The Sankey diagram provides an overview of the structure of the competitions but it can also be used to dive into the database that has been built. Each category can be selected in order to expand the diagram revealing new curves that link each category to a collection of electroacoustic compositions that have received a prize during the competitions and are part, for many of them, of the IMEB archives.

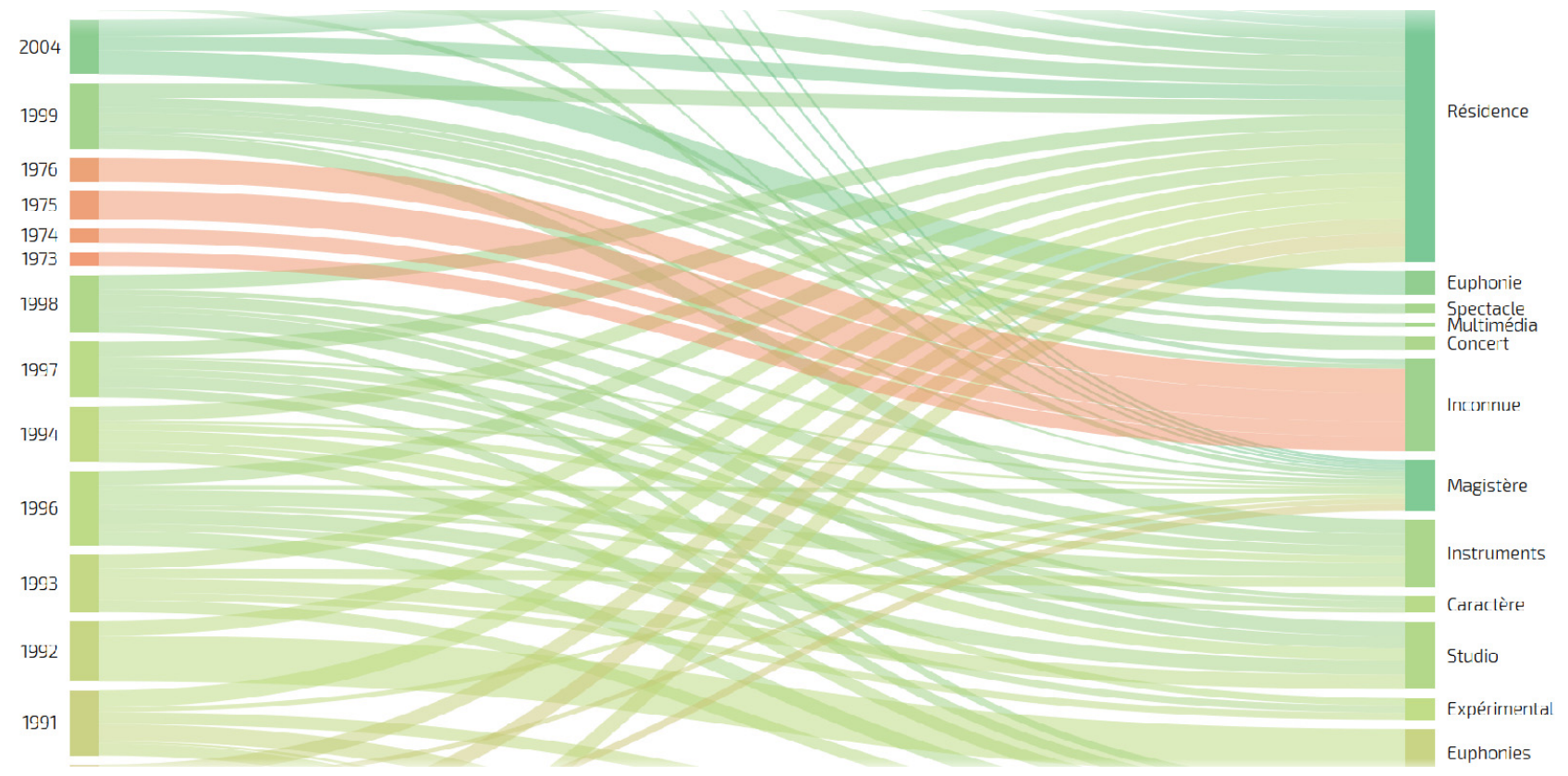

Figure 1: Fragment of the Sankey diagram used to represent the evolving set of categories. 
The flow diagram filters automatically the awardwinning works' collection based on the user inputs: only the information about the electroacoustic compositions whose category has been selected is displayed. This information includes the title of the composition and the name of its author. It can be used to access a more complete data set stored in the database that indicates for example its date of creation, its duration or its number of tracks. The main purpose of these titles collections is to give access to the electroacoustic compositions themselves to be able to listen to the IMEB archives even if this access will only be granted inside the $\mathrm{BnF}$ premises.

The online version of the flow diagram indicates the compositions that are part of the IMEB archives. They reference audio files that are stored inside 6292 digital folders made by Christian Clozier and Françoise Barrière. Named "capsule," each of these folders contains information about an electroacoustic composition that has been at least submitted to one competition or has been publicly presented during the music festivals "Synthèse" organised by the IMEB. Together, they provide a rich panorama of the actors of the contemporary musical creation of the second half of the 20th century.

Like the other interactive visualisations presented in this paper, the flow diagram references these capsules to allow its users to discover their content while browsing between different categories of the IMEB competitions. The flow diagram creates a search context and define a contextual search by letting its users explore the capsules content from the lists of award-winning compositions ordered by categories in reverse chronological order.

\subsection{Interactive Index and Line Charts}

To give an insightful representation of the IMEB competitions more than one data visualisation has been created. This plurality permits us to focus on different properties of Bourges's international competitions. The next data visualisation represents an interactive index. Unlike the flow diagram, this index doesn't highlight the competitions categories. It reveals the large number of competitions participants by referencing all the composers whose name has been found in the minutes archived at the $\mathrm{BnF}$.

For the time being, only minutes from 1973 to 1994 have been completely processed. But data has also been gathered for the editions that took place between 1996 and 2009 using the content of the 6292 capsules to identify new participation. To sum up, 2,590 different participants have already been identified and 1,202 of them have at least one of their electroacoustic compositions stored in the IMEB capsules.

The interactive index gives an overview of these 2590 participants while allowing its users to do quickly the distinction between those whose works are present in the capsules and those whose works do not appear in it: the graphical elements that are referencing them are rendered with a semitransparent colour to grant more visibility to the other graphical elements fully opaque that can be used to pinpoint electroacoustic compositions that are part of the IMEB archives.

The interactive index associate each identified composer to a grey square followed by at least one coloured square (see Figure 2). The number of coloured squares indicates the number of competitions each composer has participated while their colour represents a specific edition. The red ones represent the first edition of the competitions organised in 1973 while the purple ones point out the last edition that took place in 2009. The colours go from red to purple passing by orange, yellow, green and blue. They allow to quickly identify composers that have participated in more than one competition but also the ones that have contributed repeatedly to the activities of the IMEB between 1973 and 2009.

The interactive index invites by default to access data about composers that have participated multiple times to international competitions and have at least one of their electroacoustic pieces stored in the IMEB capsules. These composers are represented by several coloured squares that creates linear colour gradients that can be rapidly identified. The longer these gradients are, the more chance they have to pinpoint to composers whose works have been particularly well preserved. Among these composers we find Eugeniusz Rudnik, Åke Parmerud, Francis Dhomont, Jonty Harrison and Maggi Payne. All have participated in different editions and several of their electroacoustic pieces are part of the IMEB archives. To go into details, one hundred capsules out of 6,292 are dedicated to their works and each one of them contains several documents (mainly audio files and notices) about a unique composition. 


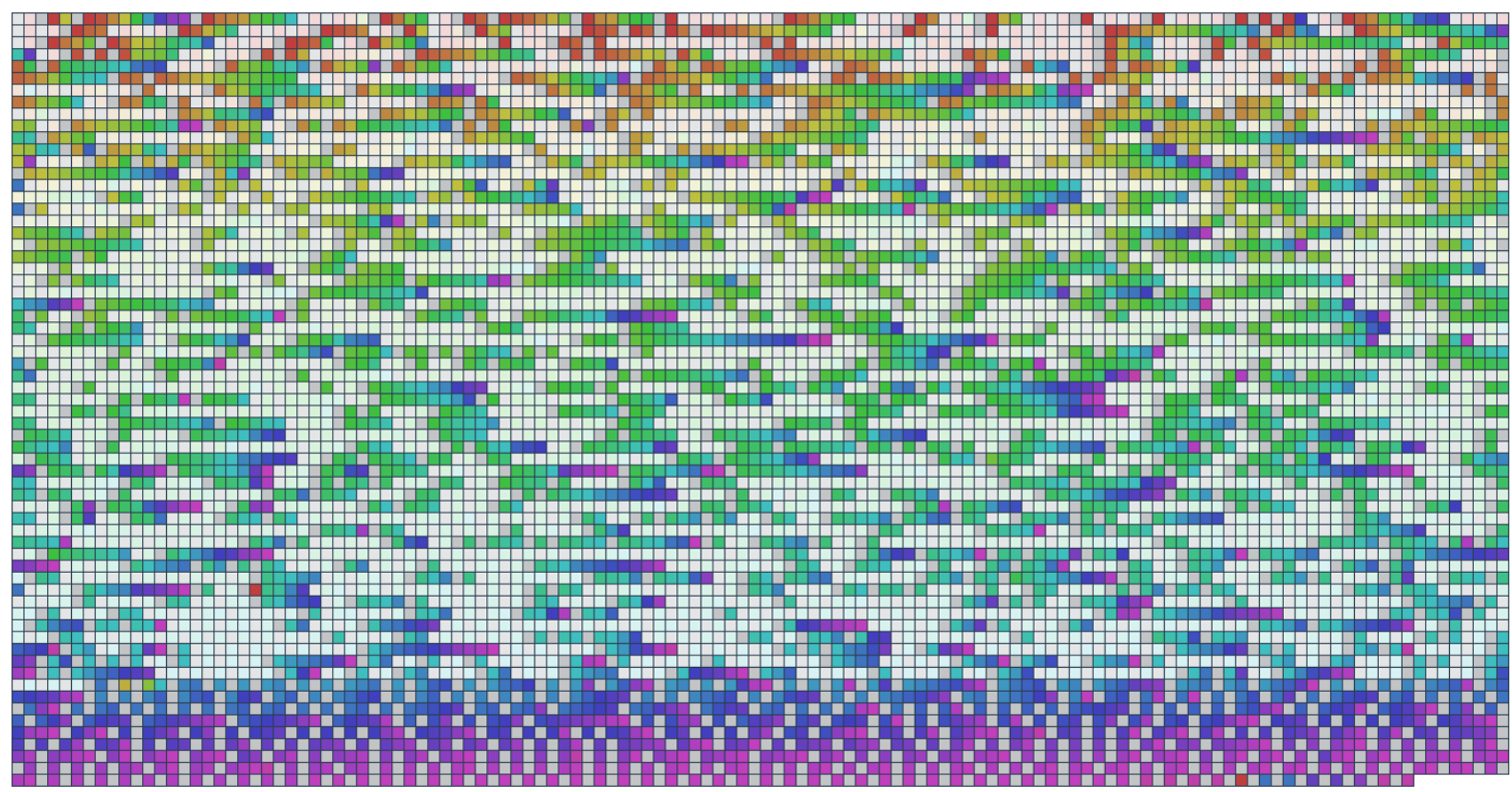

Figure 2: Interactive index used to give an overview of the 2590 identified participants.

Like the flow diagram, the interactive index proposes an overview of the international competitions using one of its main properties: the number of participants. But it can also be filtered using a search bar. The search bar lets highlight a specific composer using their name and first name in order to retrieve information about all of its compositions that are present in the IMEB capsules. The interactive index follows the "seeking mantra" defined by Ben Shneiderman to explore digital collections: "overview first, zoom and filter, then details-on-demand" (1996). When selected, each series of coloured squares give access to information about a different composer. This information includes the number of participations and their year. It also indicates the nationality of the artist and the list of all its electroacoustic compositions that are present inside the IMEB capsules; a list that can be used at the National Library of France to listen to them.

The menu of the interactive index can also be used to filter the index in order to display for example only information about composers that have participated in one or more editions chosen by a user. The interactive index lets its users retrieve data about a specific composer using advanced search criteria but it also permits its users to explore the content of the IMEB archives by selecting several of its graphical elements based on their position and colour without having to know necessarily any composer's name. The interfaces presented in this paper are designed to highlight by default the main actors of the IMEB competitions and reference the documents that are available at the $\mathrm{BnF}$.
Another type of interactive visualisations has been designed to continue to explore the IMEB archives without having to know in detail the history of electroacoustic music. These interactive visualisations are line charts that let their users access the information stored in the database by country. The line charts show the total number of participants by countries for each edition based on the information contained in the competition's minutes. They give a clear picture of the increasing participants number, edition after edition, from 1973 to 1994. The minutes of the editions that took place between 1996 and 2009 haven't fully been processed yet but demonstrates the competitions' success by reporting each year more than 500 candidates' applications on average.

Compared to the minutes, the line charts don't indicate the amount of candidate's applications but the total number of participants (who sometimes submit more than one electroacoustic composition) in order to be able to follow more easily the evolution of their number but also to know how many composers are from a specific country. By default, the line charts provide information about all the countries that have been identified but each country can be individually selected to retrieve the list of all the composers that share the same nationality or live in the same country. These dynamic lists highlight composers' name whose electroacoustic pieces are referenced in the IMEB capsules and indicate the number of them that can be listened to. For example, 179 composers from the United Kingdom have been identified and among them 91 are referenced in the IMEB 
capsules who give access to 361 of their electroacoustic compositions.

The interactive visualisations that have been previously described don't pretend to grant access to all the information related to the IMEB competitions that have been archived at the BnF. On the contrary, they try to give an image of the archives where the information still missing is represented as such. Like the database they are using, the interactive visualisations can always be edited and enriched. They represent navigation tools that allow their users to browse the IMEB capsules while providing overviews and references to the BnF general catalogue. Theses overviews focus on different properties of the electroacoustic competitions in an attempt to facilitate access to the records of the electroacoustic compositions while linking to other resources that are available and share some of their attributes. The last section of this paper describes another method to create dynamic links between information nodes based on navigation paths and user inputs.

\section{VISUALISING NAVIGATION PATHS}

In order to extend the accessibility of the IMEB archives a multi-agent system has been designed (MAS). This system is used to draw maps that represent the data has been retrieved from the database by a unique user while using the interactive index presented previously.

Each agent of the MAS is created from an information node related to a composer or an electroacoustic piece. The information node represents its genetic string. It determines its appearance (colour, shape) and behaviour (movement speed, actions). It allows them to appear with different colours and behave differently.

Like fireflies, each agent has the ability to interact with other agents that are spatially closed to him. These interactions serve to create group dynamics. They are used to automatically compare information nodes and reveal the properties they have in common. Then, the list of properties that are shared by most of the agents can be used to reorganise the whole system. They became part of the graphical interface allowing its users to choose between them in order to create new grouping between agents.

The main characteristic of this type of system is their ability to gradually evolve in order to show in real time the main attributes of the information a user is accessing. It adapts to an ever-changing environment.
The first version of the MAS that has been created progressively regroups by countries all the nodes that referenced an electroacoustic music composer. First, the system's agents interact between them to determine the attribute they have in common. Then, they choose to regroup with the ones having for this attribute the same value as them. They give birth to a new agent that represents this attribute and value: a country like France or Italy, for example. Each of these new agents represents a common point between at least two other primary agents which become its child nodes. This transformation and not disappearance of nodes that share a common attribute associated with the same value lets us keep track of all the nodes that have been consulted while regrouping them. Any node that becomes a child of another can be reached as well as the information it transports by selecting its parent. In other words, the MAS allow its users to retrace their path and discover new properties that are shared by the information nodes they have consulted.

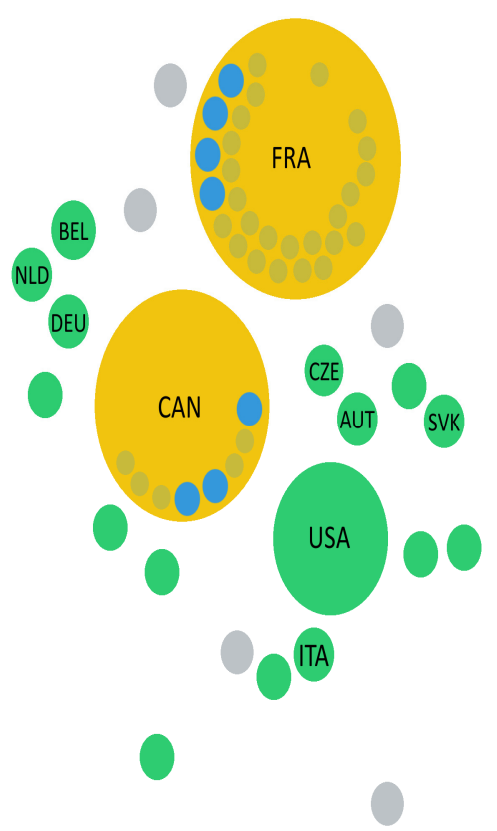

Figure 3: Example of groupings made using a MAS.

Figure 3 shows parent and child nodes both. The grey elements are nodes of information sharing any attributes with the other ones while the green nodes are grouping of several nodes of information that can be opened to discover each of them. The yellow ellipses are parent nodes that have been opened to access their child nodes.

While being associated with the interactive index, each system's agent can represent either a composer, a country or an audio composition and can be grouped accordingly. But the nature of each element is only one of many variables that can be 
used as a common denominator to gather several agents. The MAS can also be used to order awardwinning composition by categories (abstract music, program music, electroacoustic music with instruments, sonic art work, etc.). This possibility appears automatically once the information about several electroacoustic compositions belonging to the same category has been accessed.

By generating multiple groupings between agents based on a common attribute chosen to display them, this kind of interactive visualisation allows a user to acquire knowledge about its click stream. MAS are a powerful tool for exploring databases. They let us build graphical interfaces that are able to evolve gradually with a minimum of user input and knowledge about the content and structure of a database.

\section{CONCLUSION}

The different data visualisations presented in this paper aim to facilitate access to the IMEB archives located at the National Library of France. They focus on the electroacoustic international competitions organised by the IMEB from 1973 to 2009 and provide different ways to access information about their participants and audio compositions that have been submitted to enter the competitions.

The information that can be retrieved comes from different documents available at the $\mathrm{BnF}$ like the competitions minutes or data collections like the 6292 digital folders made by Christian Clozier and Françoise Barrière to represent the diversity and richness of electroacoustic music presented during the IMEB festivals and competitions.

Together, these interactive visualisations represent an attempt to create interfaces that can display some of the specificities of the IMEB archives but also connect its content to other resources like the $\mathrm{BnF}$ general catalogue using a database that references its entries about electroacoustic composers. In other terms, they represent an attempt to provide interfaces that encapsulates the IMEB archives specificities while trying to open its content to new networks, to new forms of visibility and browsing as the multi-agent system used to visualise navigation paths shows it.

\section{ACKNOWLEDGMENTS}

We would like to thank Françoise Barrière and Christian Clozier for their entire lifework and insights about the IMEB activities. We are thankful to the Audiovisual Department of the Bibliothèque nationale de France represented by Audrey Viault and Pascal Cordereix, as well as the Department of Music and its Conservation and Communication service represented by Catherine Vallet-Collot. We would also like to extend our thanks to Associate Professor Geneviève Mathon and research assistant Azadeh Nilchiani for their feedback and support.

\section{REFERENCES}

Cruz, P. and Machado, P. (2011) Generative storytelling for information visualization. IEEE Computer Graphics and Applications, 31(2), pp.8085.

Ferber, J. (1995) Les systèmes multi-agents: Vers une intelligence collective. InterEditions, Paris.

Manovich, L. (2002) The Language of New Media. The MIT Press, Cambridge, Massachusetts.

Manovich, L. (2011) What is Visualization? Visual Studies, 26, pp.36-49.

Shneiderman, B. (1996) The Eyes Have It: A Task by Data Type Taxonomy for Information Visualizations. In Proceedings of the 1996 IEEE Symposium on Visual Languages, Washington, DC, USA. IEEE Computer Society.

Whitelaw, M. (2015) Generous Interfaces for Digital Cultural Collections. Digital Humanities Quarterly, 9(1). 